\title{
Intelligent Robotics in Smart-Factories during the Fourth Industrial Revolution
}

\section{David Avishay, Phd $^{1 *}$, Veselin Pavlov, Phd $^{2}$, Galia Pavlova, Phd $^{3}$ and Guy Kashi Phd $^{1}$}

${ }^{1}$ Department of Industrial Management and Engineering, Azrieli College of Engineering Jerusalem, Jerusalem, Israel

${ }^{2}$ Automatization Faculty, Technical University of Sofia, Sofia, Bulgaria

${ }^{3}$ Computer Systems and Technology Faculty, Technical University of Sofia, Sofia, Bulgaria

\begin{abstract}
The application of intelligent and colubrious robots has its own place in evolved industrialization. These are smart factories and plants. It seems insufficient to rely on modern fast and accurate automation and robotics. Today, science looks at the forms of production management. There are reserves to improve the quality of production, with reduced financial costs, time and energy. The security, stability and safety of production are set to desirable peaks that are unattainable today. To achieve this, appropriate flexible production schemes are needed, tailored to the specifics of production. In the present work, a model suitable for universalization and intellectualization is considered according to the latest scientific, digital, technological, discoveries, human biology and machine training and self-learning.
\end{abstract}

\section{Keywords}

Robot, Robotics, Artificial intelligence, Management of production, Smart factories, Intelligent technological schemes, Industrial revolution 4.0

\section{Introduction}

In order to understand the task in depth is important to measure two global dimensions:

1. Historic base and granted knowledge at the moment. We should not also miss the gathered engineering, technological and management experience, gathered until today.

2. The biological and physical restraints, that nature imposed on us. In this we include the physical and biological abilities of the human and animal world with their specifics and wondrous possibilities.
The automatization and the robotics are not completely fulfilling their tasks during the Third industrial revolution. The role of logistics in production is exhausted. We used even the last reserves of the smart human management.

The role of intelligent management through $\mathrm{Al}$ in the industry is becoming imperative. This new management is needed to be seen through the existing at the moment conflict between the human and the artificial intelligence.

Where in the modern, automatic smart production

*Corresponding author: David Avishay, Department of Industrial Management and Engineering, Azrieli College of Engineering Jerusalem, 26 Yalov Shraibom, Jerusalem, Israel

Accepted: September 21, 2019; Published: September 23, 2019

Copyright: (c) 2019 Avishay D, et al. This is an open-access article distributed under the terms of the Creative Commons Attribution License, which permits unrestricted use, distribution, and reproduction in any medium, provided the original author and source are credited.

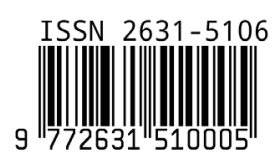

Avishay et al. Int J Robot Eng 2019, 4:017 
we can fast and easily introduce AI?

1. The intelligent marketing.

2. The intelligent design and the intelligent production technology.

3. The intelligent supply.

4. The intelligent production preparation (and ensuring uninterrupted process). The intelligent production, reserves consideration.

5. The intelligent support and maintenance of the process of production. The intelligent definition of quality and quality management.

6. The intelligent forming and packing of the final product.

7. The intelligent storage in intelligently organized intermediate warehouses. The intelligent delivery into the commercial places.

8. The intelligent accounting of production and delivery processes and intelligent update of the systems.

9. The circle of the process with intelligent changes into repeating cycles.

10.The intelligent analytics of the whole process - from the marketing to the client's receiving of the product with consideration of the reserves.

11. The intelligent assessment for usage and liquidation of the product, when it is not needed anymore.

Of course we can interchange the word "intelligent" with the word "Smart". In fact the artificial machine intelligence can pretend for higher place of activity and be similar to human intelligence, soul or religion for automatic production.

In order for a new term to find its own place it is necessary to make one explanation. The intelligent or smart production is one of the functions of the smart factories. The smart factories are created so they can fulfil the necessity of full utilization of the reserves of production with minimal loss of materials and energy, without polluting the nature and without adding and creating technological conflicts. This means that everything - from the administration and designing activities to marketing and deliver of the product should be subjected to logistical smart algorithm in the form of artificial intelligence [1].

\section{Universal Scheme for the System of Smart Production}

With the presented here universal scheme for the system SOFI), responsible and engaged with the pro

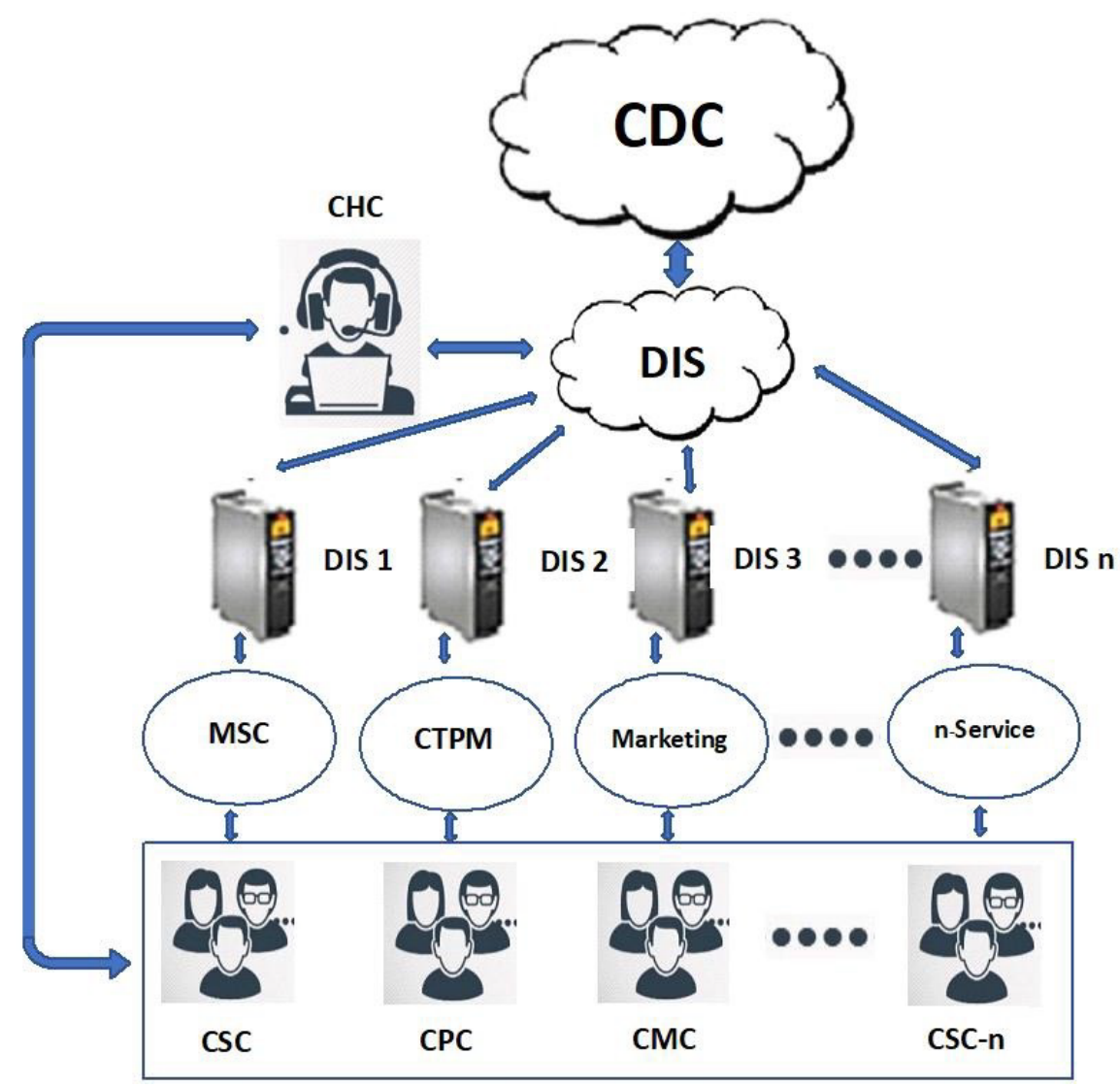

Figure 1: Universal scheme for systems (SOFI). 
duction in smart factories and those with intelligence logistics, is aimed a bigger efficiency to be created with minimum of physical costs (Figure 1). This scheme has flexibility, universality and completion. It is based on research of different other schemes, researched and applied in the world [2].

The universal scheme SOFI (Smart Organization Factory in Industry) is made of: CDC - Cloud Data Cluster; DIS - Differential Intelligent Service; CHC - Collaborative Human Control; MSC - Manufacturing Service Control; CTPM - Clean Technological Process of Manufacturing; CSC - Collaborative Support for Production; CPC - Collaborative Production Control; MC - Collaborative Marketing Control; CSC - Collaborative Service Control; IWS - Integrated Work System; CIWS - Collaborative Integrated Work System.

The application of the scheme in different places of production and service is making it universal which gives possibilities for adding and removing different factors from the scheme and that is her functionality and flexibility. The possibility for direct application is making the scheme complete. The place of flexibility in DIS (differential intelligent service), which allows moving and coordination for the needed and specific for the production information, is very important.

The scheme is created with compliance with the biological structure of the human body that will allow the functioning of the commanding logistical operations of the specific organs. The human brain is managing with its commands everything that is happening in the body and it is helped with the other managing organs which are located in different places in the body and are asking the body to perform different activities movement, digestion, activities of the different glands, sexual activities, excretory organs etc.

In this system the CDC is the main information and managing part. Its role is to distribute the different functions to the helping and informational parts DIS who are under permanent collaborative control of their activity by CHC. DIS is also giving commands and logistical applications to the different processes of production (MSC, CTPM, Marketing etc.), which are also under separated collaborative control (CSC, CPC, CMC etc.). Thus a permanent informational and controlled flow is created without creating conflicts between the different parts. A progressive, logistical

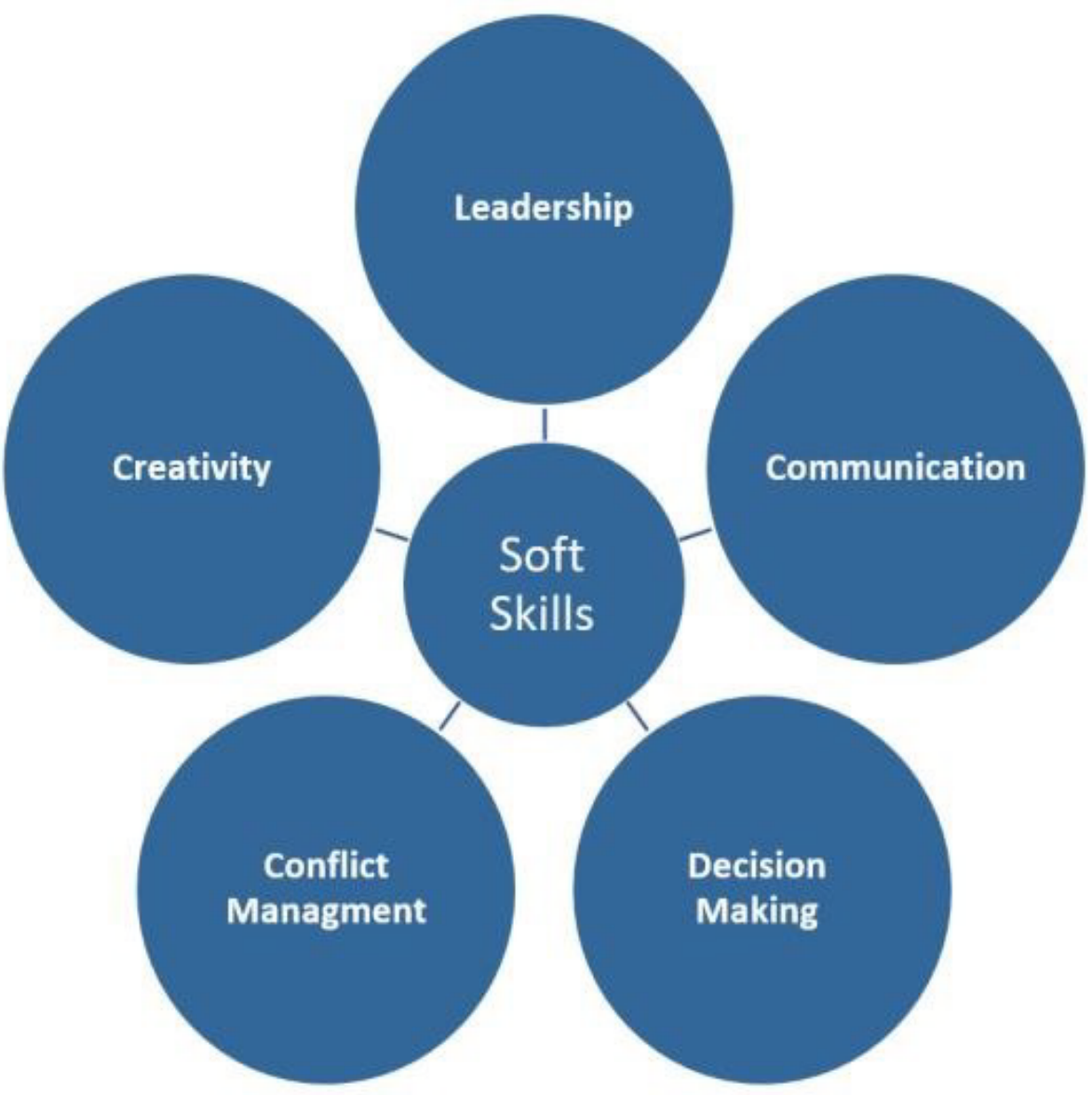

Figure 2: Soft skills is stabilizing the position of education, abilities and knowledge of the modern human, using the digital technologies. 
and controlled artificial intelligence is used, with focusing on the final goal without subjective obstacles.

\section{Soft Skills}

Soft skills is modern term that is stabilizing the position of education, abilities and knowledge of the modern human, using the digital technologies (Figure 2).

All of the skills that are not connected with the concrete specialty like leadership, communication, ability for team-work, conflict resolution skills, trust building, time management, decision making, negotiation skills, etc. are called soft skills. Those skills are harder for measure but do not require special qualities that are based on talent and are easy to be replaced by artificial intelligence. For everyone who is working in groups those are very important qualities.

The replacement of human with robots with intelligence and soft skills is very suitable in a repeated tasks, cycles, answering questions with bot machines, avoidance of conflicts and collaboration.

The concentration of information in the powerful modern servers and the grouping process of that information can fully replace the need for soft skills in the production process, due to working intelligent software.

\section{Scientific Approach - Cause and Effect}

Prof. David Khayat in his book "Research of truth: You will never be afraid of cancer again" [3] is showing the reason for creation of cancer cells in human body and how to manage your body so it can work by itself with the situation and to fulfill its goal - longer and healthier life. According to prof. David Khayat the human microflora is in danger of creating more bacteria than cells. For better functioning of the body, our immune system creates antibodies (proteins, created by leucocytes and delivered through the blood vessels). For those antibodies to work properly they need information for the type of the threat. This genius immunotherapy is absolutely applicable to the modern smart production and here is the place of the artificial intelligence with the corresponding intellectual power. Banks with the necessary information through cloud technology can recharge the programing "antibodies" that can find deviations in the process and to give information to the self-learned process control. The lack of subjectivity in this procedure is adding for the quality of the control-executive activities.

In the realization of those activities we can follow the principles of prof. Hyatt. In the human body we can find cells, called macrophages, who when receiving the correct information, can apply the security system that they are working for. The microphages work like a police of a despotic regime. They are checking the identity papers of all of the cells and their identity password. If they didn't recognize the password, the microphages are tearing them up and they are declaring alarm to the lymphocytes. This programed check and attack can be applied in a process, controlled by artificial intelligence.

Despite this strong and effective system of despotic control, the cancer cells can get into the human body due to different tricks, which are based in the cellular intelligence. This intelligent disguise is possible even in the most organized system for production. Here we can assess the level of intelligence of the program of artificial intelligence. If she is able to find and isolate the errors, then the usage of the reserves will be successful. We should look for smart application. The intelligent errors will self-learn and will gain experience. They will learn how to put to sleep the programing "microphages" that are looking for them and to start to run wild in a place without control. This is the reason for self-control and additional external control. This is the task for the collaborative human control into the SOFI system. For full effectivity, the collaborative control should be used on horizontal level for every other activity of production and as whole additionally to control the controlling systems by a collaborative part in higher level. Here more important is the role of the intellectual experience and not of the knowledge and habits.

\section{Robots with Intelligent Machine Conscious- ness}

In terms of genetics the human being and the animals are very close and in some identifications even similar and that is why the results of experiments over animals can be applied on humans in different spheres - from creating medications and stopping the aging process to forms of group management (in herds, flocks, etc.). One of the important things in artificial intelligence, put in the modern robots is to explore and report the used materials, which is faster than with the biological beings. The tiredness of the material is very fast and in some cases instantly. The reason for that is that the machine is not able to develop itself for better results or to keep the present situation (the human can do sports) and cannot message in advance for future break of activity (the machine is not getting sick or having pains). For their condition we judge based on external or internal characteristics and experience.

In the next level this is the programing of intelligent machine consciousness into the robots. Latest experiments and examinations are creating neuron network and search algorithm, which is having a module for logistical search in the system in the branched tree of possibilities. Here the most successful application is the 
product Alpha Zero [4] from the Deep Mind company. In its beginnings the neuron network is very stupid. It made its predictions based on chance. The genius of the program is that it is self-learning using access to different informational banks (clouds) and to educate itself very fast. That is the plus of the collaborative production without violating the principles of competition.

In fact the common production neuron system is suggesting help at every stage and monitoring the differential tasks, leading to common goal.

As a conclusion - to be able for a smart factory to be created a necessary planning and designing is needed, and those should be very different from the used at the moment technology, network and staff.

How Industry 4.0 can Increase the Productivity with the Help of Robots with Artificial Intelligence

In the basis of the automatized production in the time of the third industrial revolution is the industry robots. In the fourth they continue to be important factor but their activity is changed due to the new functionality. In the beginning the usage of collaborative robot-helpers will be used until the moment where a secure and stable internet network start functioning. When the network secure the action of the system, the main functions will be held by self-learning robots, using artificial intelligence.

Internet will give the necessary information, based on the commercial research of the market and will upgrade intelligently the process of production while minimizing the reserves.

How to use those advantages for upgrading the process of production? In the world's scientific literature we see the introduction of new terms [5]:

OPE - Overall Production Effectiveness

OEE - Overall Equipment Effectiveness

OLE - Overall Line Effectiveness

SMT-Surface-mount Technology

KPI - Key Performance Indicator

How to determine when will have readiness for organizing a smart production. Despite the fact that the expected results have positive value for the quality of the production and the staff, this production won't be effective if it is not designed in a smart way. And to be effective it needs to follow the following equality:

OPE $=$ [availability of equipment $] \times$ [indicator for quality performance]

For example - for one of the most-advanced modern production - of printed circuit boards (SMT) the whole production process is characterized with the indicators for OPE and OEE. As opposed to OEE and OLE, the overall effectivity OPE is viewing the whole production process including not connected processes that can be put on a level of a machine or line.

If we add the term KPI for the available production equipment, the delays and the reasons for them are listed in a form directly into the memory of the machine or the system. The collaborative operator (human or machine) can find in what aspect is the error and to write a comment. This information is processed by the logistical production artificial intelligence, who can make decision for the best effectivity of all the machines. The overall machine processing is giving a real picture of the real condition of the equipment and it is minimizing the time of taking managerial decision.

KPI for the effectivity of the quality can be defined through the contrast of the empirical data for the production time to the real time. The gathered information can be used directly as a basis for future investments.

When applying the empirical experimental methodology for gathering information we can use the cycle of Adrian de Groot (Figure 3). In this cycle we use Observation - Gathering and organizing of empirical facts and forming a hypothesis; Induction - Formulating a hypothesis; Deduction - Conclusions about the consequences of the hypothesis through verifiable forecasts; Checking - Checking the hypothesis with new empirical material and Evaluation - Evaluation of the test of the checking.

The distinctive feature of the scientific methodology for gaining knowledge is that this methodology is systematic and defined. The justification on scientific knowledge is happening through the empirical methodology of gaining facts - through conclusions about the truthfulness of a theory, based on facts. In this case we are speaking of gathering of real informational data.

The effectiveness of real project for smart factory is very important but for getting to that effectiveness it is necessary to take into consideration other parameters and their influence on the process:

TypeAl - Type Artificial Intelligence

MI - Machine learning

II - Initial Investment

Hc - Human control Factor OpSt - Open Standard clss - coordination Issues $\mathrm{Hr}$ - Human resources

Is - Information security

When we put those factors in mathematical modulation, they look as follows: 


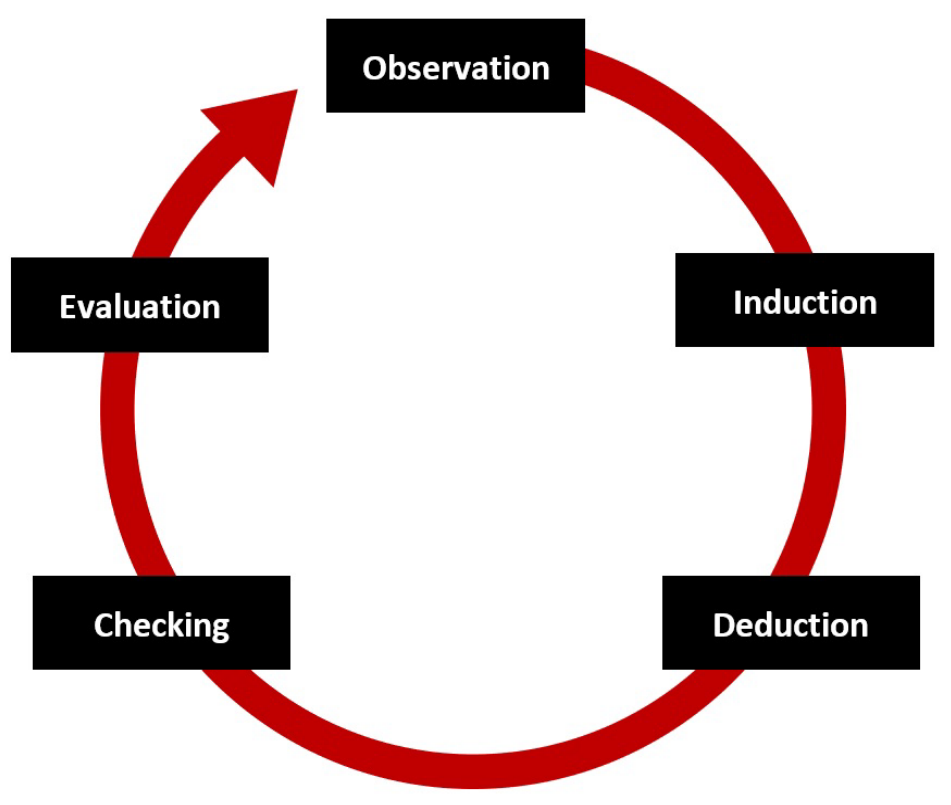

Figure 3: Cycle of adrian de groot.

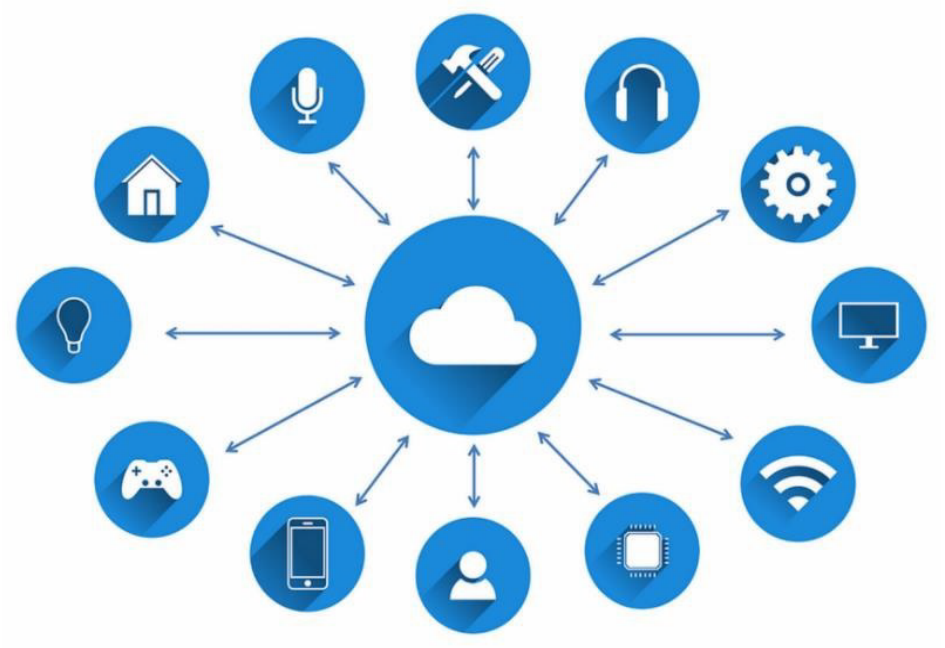

Figure 4: Digital-intellectual work and its production, based on the information from cloud technologies.

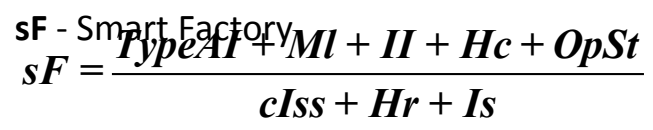

Creation of effective smart factory is depending on the positive implementation of artificial intelligence, the machine equipment, the initial investment and the control from the human factor (collaboration). From the other side the obstacles that are needed to be overcame dynamically are the coordination the production phases, the lack of educated human resource and the informational uncertainty.

The informational uncertainty is the most fickle element as it is very hard to control. There is uncertainty in the stability of the work. Every stop or slowing of the speed can create to a situation where production is impossible. The transformed power of the technology is compared to a turning points like the general electrification. The reaching of stable electric system took dozens of years to humanity. From the other side the possibility of getting fake information is big. In the project for smart factory in that area we should be working systematically and using the most advanced technological discoveries.

\section{Is Industry 5.0 near?}

The human creativity always will be important factor, but the changes should be compared to the popularized innovations. The education at the moment is not acquit to the expected technological future. This unfavorable conclusion is done by statistics all around the world. The fast development of the artificial intel- 
ligence and the integration in more aspects of life will need new equipment, program management and networks. This will make the challenge for the human to be educated and psychological ready for the caprices and the pleasures of the artificial intelligence future, bigger. Automated transportation, digital control of energy, robotization of medicine and education will be the basis of the next technological revolution. But the most important - the usage of artificial intelligence will be seen the most in industry, which will be forced by the conditions of time to produce faster, more accurate, with better quality and with fewer costs. From other side the whole way of the product - from production to the client will be through machines, and the cost for commissions will drop and the prices will be lower.

The amplitude between the price of machine-intellectual industrial production and the hand-made products will be sensible. The people will be interested in the digital- intellectual work and its production, based on the information from cloud technologies (Figure 4).

\section{Application of SOFI}

SOFI is virtual project that contains few horizontal projects that can be developed in the different levels of the scheme (Figure 1). This means that the scheme is applicable if few conditions are met:

1. In the scheme a smart algorithm (smart logistical and technological sequence) is working - a sum of rules who are given to the computer programs and they need to follow them unquestionably.

2. Applicable artificial intelligence with private orientation - this is intelligence that is created artificially and specially for the private production. We don't need to universalize it and to go with applications outside the specific productions.

3. Deep learning - machine learning, designed to have more levels and nuances and based on the human brain. The deep learning is possible through two key technological advancements - more data (information) and better hardware.

4. Machine learning - the next level of programing of the software and the hardware in which the machines can do self-learning.

5. Neuron networks - imitating the processes in the human brain, connected with the idea of deep learning. The development of neuron networks in the last years is possible due to the processors of high class.
The presence of those conditions can lead to technological implication which means that everything written in the scheme is possible and the conditions for it are met and the environment is ready for implementation. It is met the criteria for applying the scheme at the right place (factory, production place) in the right time (science and society are ready for the next revolution).

As an addition the informational stability and security will get better using the new generation of wireless mobile networks $5 \mathrm{G}$ which will help the usage and service from distance - from home or from different place in the world.

\section{Conclusion}

The smart factory is not the one which is led by smart director or have smart management. For industry 4.0 that is not enough.

In fact a combination of different technologies and solutions that can be integrated together in the production eco-system will result in smart production. That is the first step to smart factories. In this case the role of eco system is done by the Industrial Internet of things (IIoT). One of the most important factors is the artificial intelligence. The search and application everywhere of Al has supporters and critics. Bill Gates is comparing the artificial intelligence with the nuclear energy and the nuclear weapons and how the same thing can be used for the good of the society or be dangerous to the society. Gates say that the artificial intelligence can help a lot in the health system and education. Technologically it is applicable in the industrial production which will bring the application of the smart factories in our time.

\section{References}

1. D Avishay, V Pavlov, G Pavlova, B Petrov, N Dimitrov (2019) Industry 4.0 - Distributed mobility robots and intelligence elements. Global Journal of Computer Science and Technology 19.

2. D Avishay, V Pavlov, G Pavlova, G Kashi (2018) Industry 4.0 - new features for artificial intelligence robots.

3. D Khayat (2018) L'Enquête vérité: Vous n'aurez plus jamais peur du cancer.

4. G Marcus (2018) Innateness, alphazero, and artificial intelligence. New York University, USA.

5. A Bonara (2016) Calculating basic performance indicators for the smart factory: Overall Production Effectiveness (OPE).

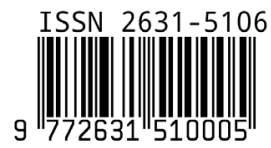

